\title{
Design, Analysis and Manufacturing of a Car Rear Spoiler for Drag Reduction
}

\author{
Dr M.V. Sathish Kumar' ${ }^{1}$, B. Ashwin Rao ${ }^{2}$, Dr G. Mallaiah ${ }^{3}$ \\ Associate Professor, Kamala Institute of Technology and Science, Huzarabad, Telengana ${ }^{1}$ \\ M. Tech, Student, Kamala Institute of Technology and Science, Huzarabad, Telengana ${ }^{2}$ \\ Assistant Professor, Kamala Institute of Technology and Science, Huzarabad, Telengana ${ }^{3}$
}

\begin{abstract}
Aerodynamic characteristics of a Honda civic car 2009 are of significant interest in reducing car-racing accidents due to wind loading and in reducing the fuel consumption. Even though these vehicles typically have a more rigid chassis and a stiffer suspension to aid in high-speed maneuverability, a spoiler can still be beneficial. In this thesis will focus on CFD-based lift and drag prediction on the car body after the spoiler is mounted at the rear edge of the vehicle, a car rear spoiler is designed and modeled in 3D modeled software CREO by different models of spoilers (NACA 4412\& NACA 6409) and analysis done by ANSYS software at different speeds (70, 90 \& $110 \mathrm{~km} / \mathrm{hr}$ ). In this thesis the CFD analysis to determine the lift force, drag force, mass flow rate, and pressure drop. 3D modeled in parametric software CREO and analysis done in ANSYS.
\end{abstract}

Key words: Finite element analysis, spoiler, CFD analysis, drag force and lift force.

\section{INTRODUCTION}

1.1 A spoiler is a component of a disseminated outline or description of any piece of fiction that reveals any plot parts that threaten to provide away vital details. Typically, the main points of the conclusion of the plot, together with the climax and ending, area unit particularly considered spoiler material. It can even be wont to confer with any piece of knowledge concerning any a part of a given media that a possible shopper wouldn't wish to understand beforehand. as a result of enjoyment of fiction depends an excellent deal upon the suspense of unveiling plot details through normal narrative progression, the previous revelation of however things can end up will "spoil" the enjoyment that some customers of the narrative would otherwise have practiced. Spoilers will be found in message boards, articles, reviews, commercials, and picture show trailers The term spoiler was introduced within the period of the net, and came to prominence in newsgroup conversations. it's still common in web articles and social media discussions. Early rules of netiquette insisted that spoilers may and will be commonly avoided, however if the posting of "spoiling" info was ineluctable, or not it's preceded by a warning like "SPOILER ALERT", or the spoiler itself must be cloaked in order that it can't be visible to any however those keen for details and not bothered at the thought of such probably plotrevealing info.

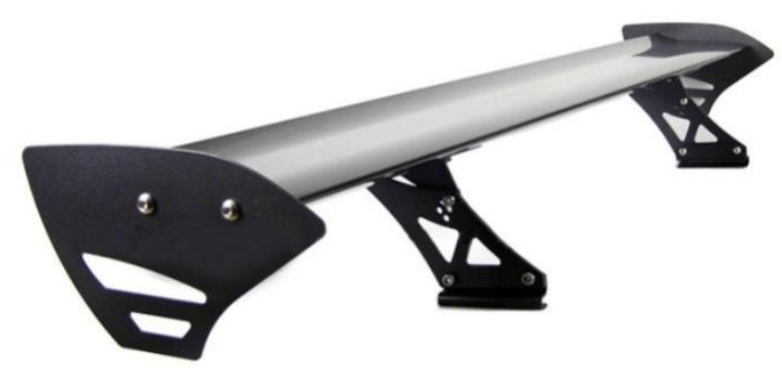

\section{Rear spoiler}

Sometimes, these warnings area unit omitted, accidentally or deliberately and a few unwitting readers have had literature, films, tv programmes and different works that they were trying forward to experiencing "spoiled". there's a typical demand, particularly among web users, to own protection against accidentally seeing material thought of to incorporate "spoiler" info, even within the web version of settings wherever such material has conventionally and traditionally appeared, like discussion teams or literary reviews. As a results of this level of objection to spoilers, trolls could post them strictly for his or her own pleasure, finding amusement in basic cognitive process they're fully destruction a narrative expertise for others. On honorable websites, these will be according to moderators and such posts taken down, the posters blacklisted, however solely once the actual fact. Most such websites give a way of 
tagging bound threads as containing spoilers for those that want to debate a fictional add depth, together with the outcomes of events and also the handling of the narrative resolution. Some have felt compelled to avoid taking part on public websites altogether, came upon "closed" websites to exclude those that area unit sensitive regarding spoilers, or determined that they had to unilaterally web log at the expense of public exchange.

\section{LITERATURE REVIEW}

Investigation of Drag and Lift Forces over the Profile of Car with Rearspoiler Using CFD

Now a days demand of a high speed car is increasing in which vehicle stability is of major concern. Forces like drag\& lift, weight ,side forces and thrust acts on a vehicle when moving on road which significantly effect the fuel consumption The drag force is produced by relative motion between air and vehicle and about $60 \%$ of total drag is produced at the rear end. Reduction of drag force at the rear end improves the fuel utilization. This work aims to reduce the drag force which improves fuel utilization and protects environment as well. In the stage of work a sedan car with different types of spoilers are used to reduce the aerodynamic drag force. The design of sedan car has been done on CATIA-2010 and the same is used for analysis in ANSYS-(fluent). The analysis is done for finding out drag and lift forces at different velocities, and spoilers. This study proposes an effective numerical model based on the computational fluid dynamics (CFD) approach to obtain the flow structure around a passenger car with a rear spoiler.

\section{PROBLEM DESCRIPTION}

The objective of this project is to make a 3D model of the spoiler and study the drag force and lift force behavior of the spoiler by performing the finite element analysis(CFD) .3D modeling software(Creo) was used for designing and analysis software (ANSYS) was used for CFD.

\section{INTRODUCTION TO CAD/CAE}

Computer-aided design (CAD), also known as computer-aided design and drafting (CADD), is the use of computer technology for the process of design and design-documentation.

\subsection{INTRODUCTION TO CREO}

PTC CREO, erst called Pro/ENGINEER, is 3D modeling package utilized in engineering science, design, producing, and in CAD drafting service companies. it had been one among the primary 3D CAD modeling applications that used a rule-based constant quantity system. victimisation parameters, dimensions and options to capture the behavior of the merchandise, it will optimize the event product in addition because the style it self .Different modules in CreoPart design, Assembly, Drawing\& Sheet metal.

\subsection{INTRODUCTION TO FINITE ELEMENT METHOD}

Finite Element Method (FEM) is also called as Finite Element Analysis (FEA). Finite Element Method is a basic analysis technique for resolving and substituting complicated problems by simpler ones, obtaining approximate solutions Finite element method being a flexible tool is used in various industries to solve several practical engineering problems. In finite element method it is feasible to generate the relative results.

\section{RESULTS AND DISCUSSIONS}

\subsection{Models of spoiler using CREO parametric software}

\section{D model}

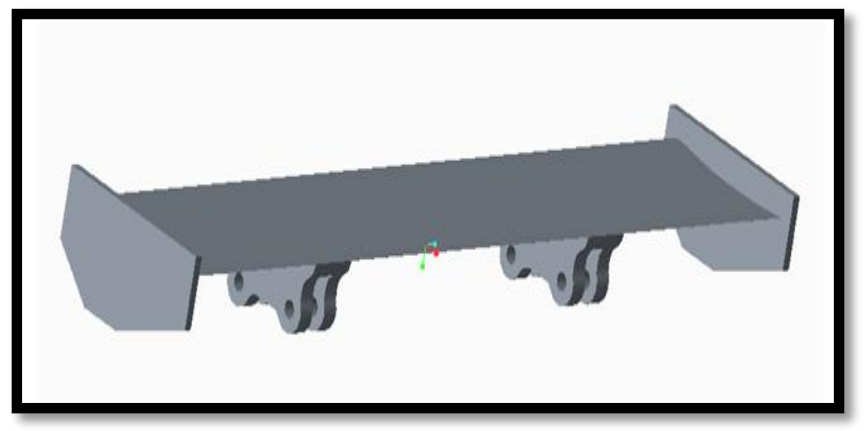


2D model

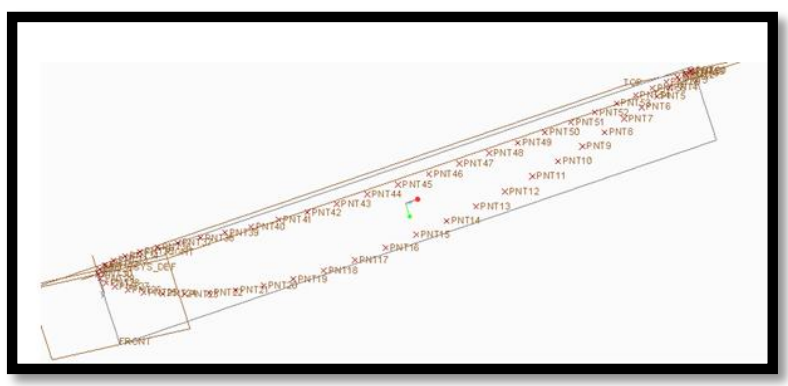

\subsection{CFD ANALYSIS OF SPOILER}

Original Model

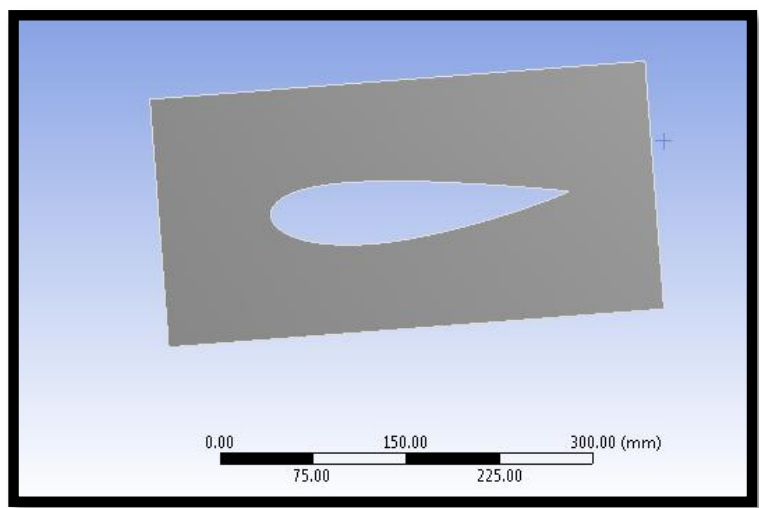

Imported Model

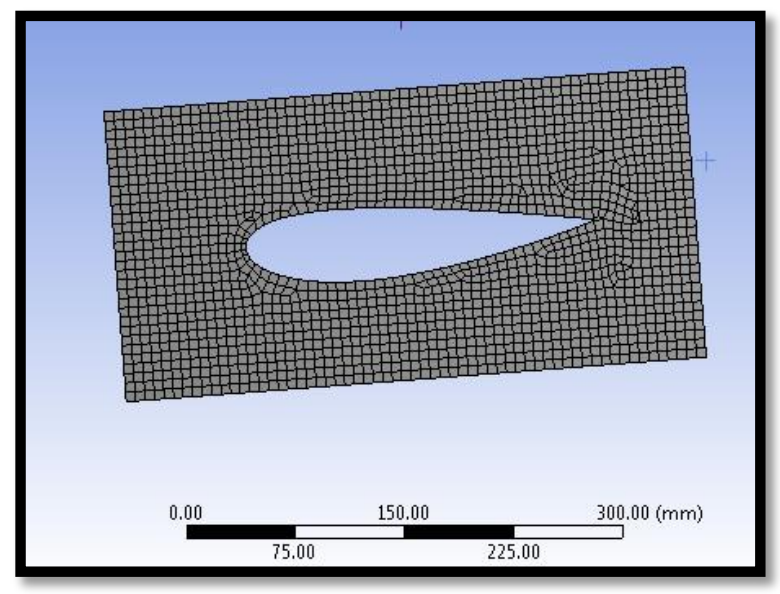

Meshed Model

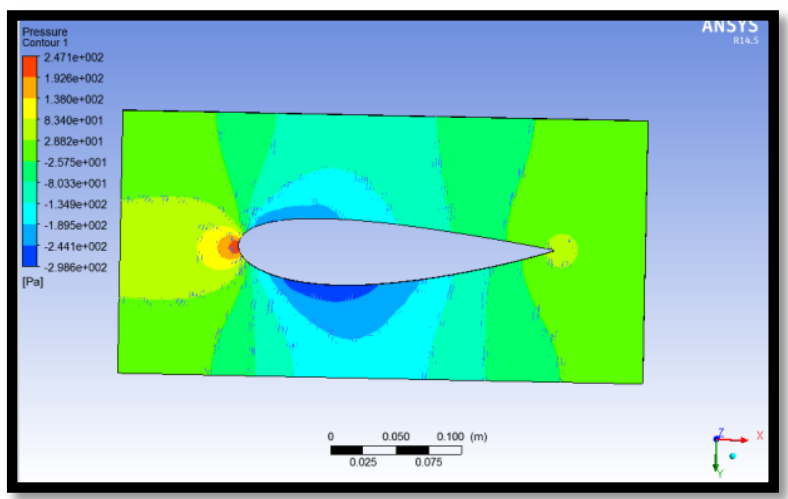


Pressure

\section{Velocity}

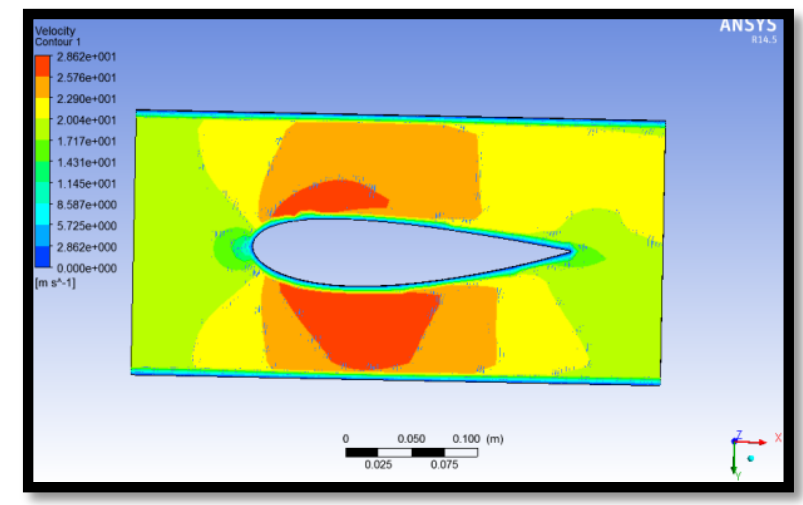

\section{DRAG FORCE}

Forces

$$
\text { Forces (n) }
$$

\section{Zone}

Pressure

Pressure

wall-_trm_srf

Viscous

(2.01518180.498861760)

(3.2900927 0.814468180$)$

Total

Coefficients

Total

\section{$0.491494450)$}

(6.26032810.802439920)

(1.8192692 -0.00736731010)

(3.834451

(2.9702354 -0.0120282610$)$

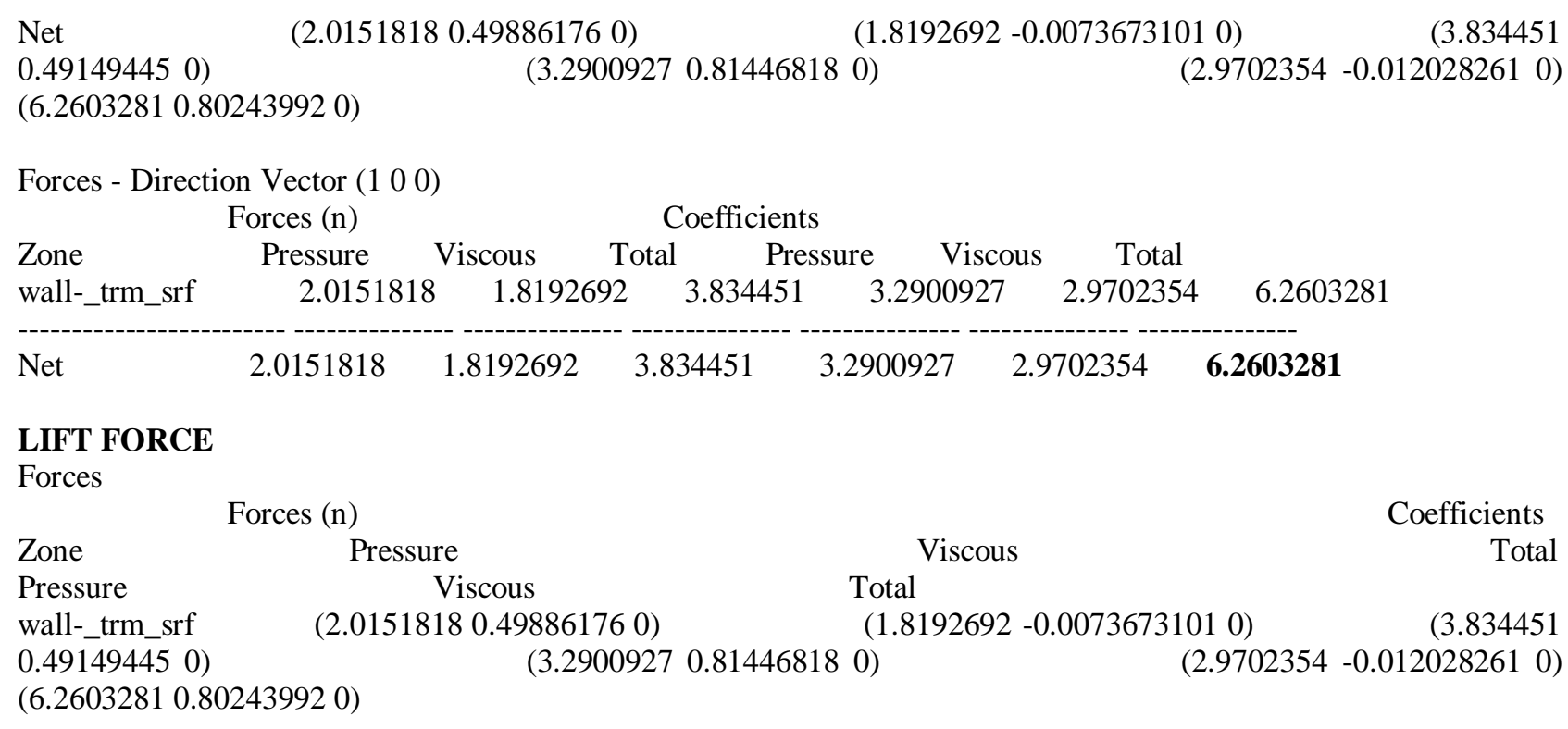

\section{Net}

(2.0151818 0.498861760)

(1.8192692 -0.00736731010)

(3.834451

$0.491494450)$

(3.2900927 0.814468180$)$

$\left(\begin{array}{lll}2.9702354 & -0.012028261 & 0\end{array}\right)$

Forces - Direction Vector ( $\left.\begin{array}{lll}0 & 1 & 0\end{array}\right)$

$$
\text { Forces (n) Coefficients }
$$

$\begin{array}{lccccccc}\text { Zone } & \text { Pressure } & \text { Viscous } & \text { Total } & \text { Pressure } & \text { Viscous } & \text { Total } & \\ \text { wall-_trm_srf } & 0.49886176 & -0.0073673101 & 0.49149445 & 0.81446818 & -0.012028261 & 0.80243992\end{array}$

$\begin{array}{lllllll}\text { Net } & 0.49886176 & -0.0073673101 & 0.49149445 & 0.81446818 & -0.012028261 & \mathbf{0 . 8 0 2 4 3 9 9 2}\end{array}$


Mass Flow Rate

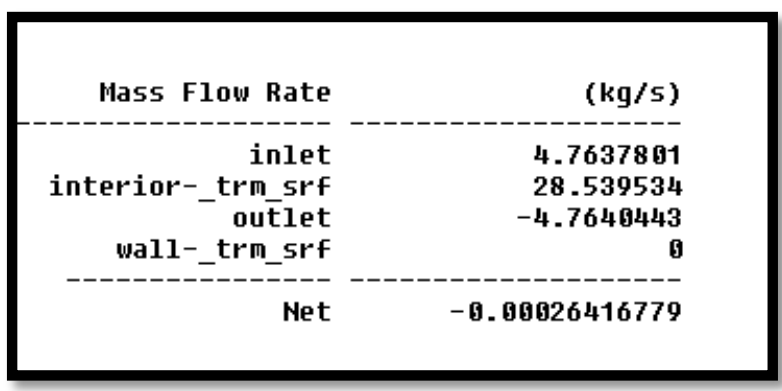

\section{MODIFIED MODEL}

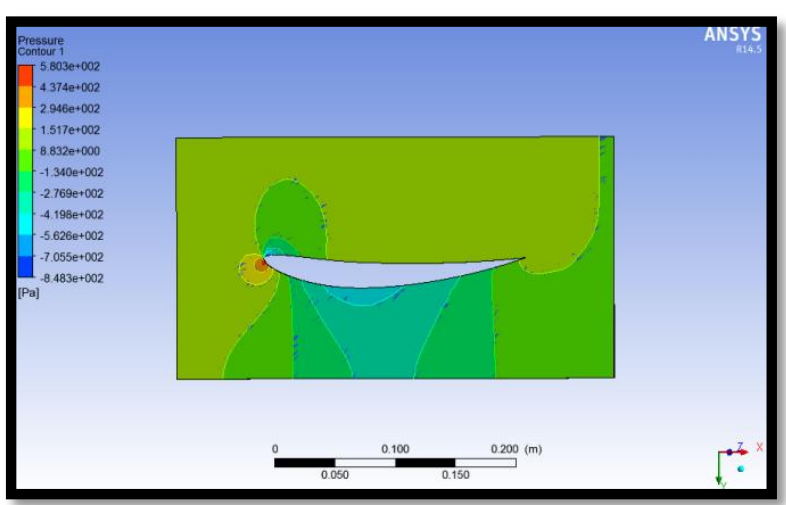

Pressure

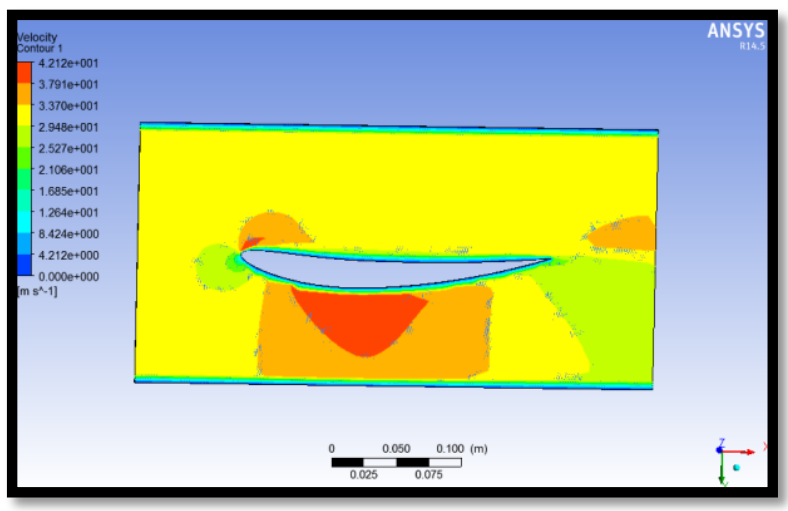

Velocity

DRAG FORCE

Forces (n)

Zone

pressure

viscous

Wall-_trm_srf $\quad(4.36819744 .47897620)$

(3.5642054-0.045592379 0)

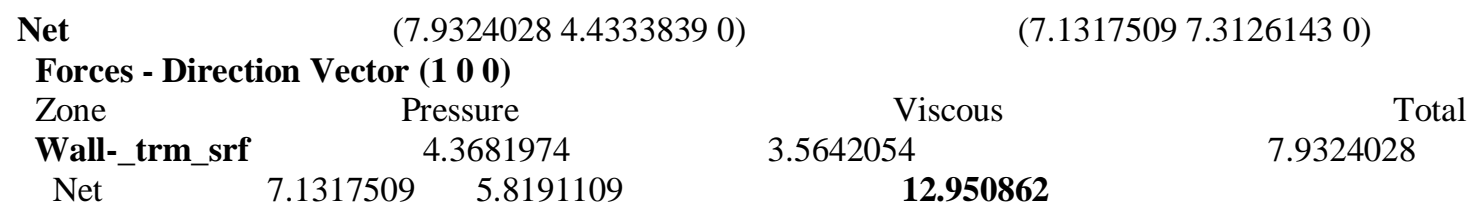

\section{LIFT FORCE}

Zone

pressure viscous

Wall-_trm_srf (4.3681974 4.4789762 0) (3.5642054 -0.045592379 0) 
Forces - Direction Vector $\left(\begin{array}{lll}1 & 0 & 0\end{array}\right)$

Wall-_trm_srf

Net

\subsection{7}

\section{Mass Flow Rate}

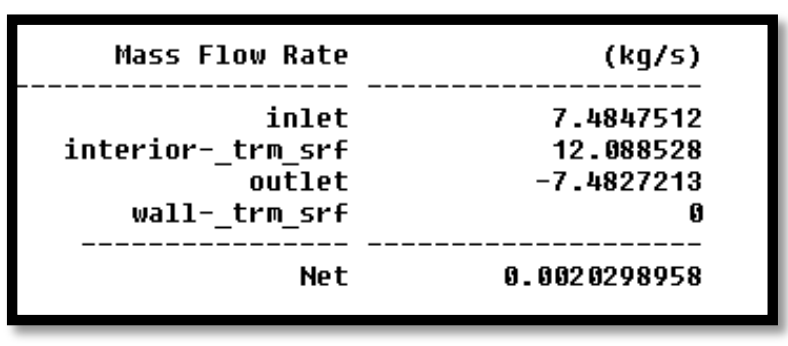

6. RESULTS AND DISCUSSIONS

CFD ANALYSIS RESULT TABLE

Original model

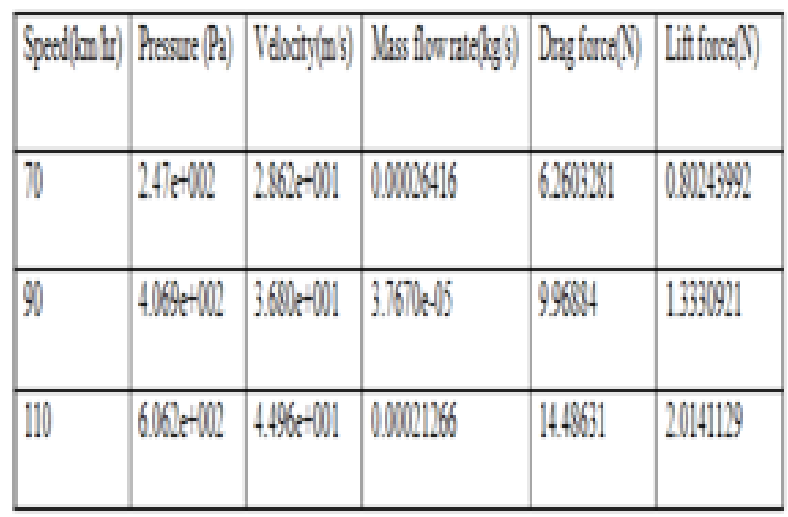

\section{Modified Model}

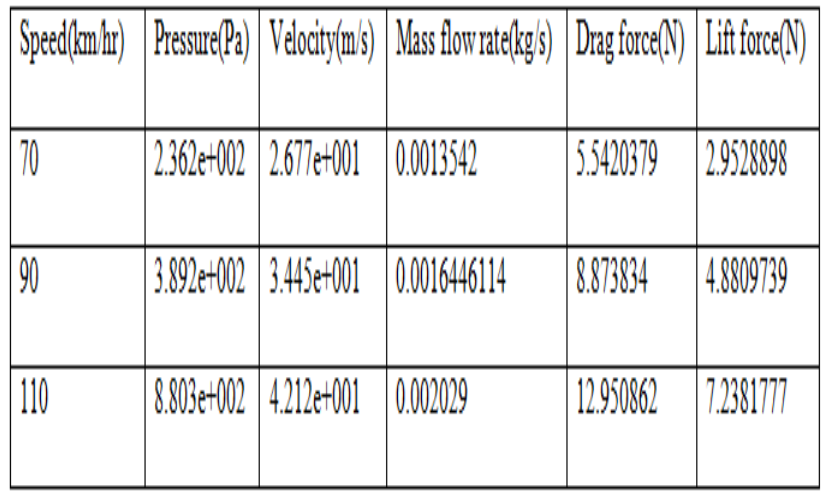

\section{MANUFACTURING PROCESS OF SPOILER}

1. A method for manufacturing an air spoiler for a automotive vehicle by blowing cylindrical raw material into a core mold and a cavity mold for manufacturing the air spoiler, comprising: positioning a plurality of separate reinforcement members into positions of said cavity mold corresponding to the area where the air spoiler is fitted on a rear side surface of the vehicle; injecting melted raw material into said cylindrical raw material, and blowing said melted raw material into said reinforcement members so as to form a first stage product integrated with said cylindrical raw material and said reinforcement members; and performing a surface finishing process for said first stage product of the air spoiler so as to obtain an end-product of said air spoiler.

2. The method for manufacturing an air spoiler for an automotive vehicle according to claim 1, wherein at the step of blowing, an engagement portion of said reinforcement member is integrally molded with said raw material, and air 
remaining in said reinforcement member is discharged through air-venting apertures formed at the bottom surface of said reinforcement member.

3. The method for manufacturing an air spoiler for an automotive vehicle according to claim 1, wherein at the step of air-blowing, said raw material is injected to integrally mold said flange portion of said reinforcement member and the air spoiler, an engagement reinforcing member is provided to assure the engagement force of said reinforcement member to said raw material of said air spoiler.

4. The method for manufacturing an air spoiler for an automotive vehicle according to claim 3, wherein said engagement reinforcing member is an adhesive tape which is adhered in ring-shape on the inside wall surface of said main body portion of said reinforcement member.

5. The method for manufacturing an air spoiler for an automotive vehicle according to claim 3, wherein said engagement reinforcing member is a predetermined plastic primer which is injected to the inside of said main body portion of said reinforcement member.

6. A method for manufacturing an air spoiler for an automotive vehicle by blowing cylindrical raw material into a core mold and a cavity mold, comprising: the steps of a) positioning a plurality of separate reinforcement members onto positions of said cavity mold, b) disposing an engagement reinforcing member into said reinforcement members positioned in said cavity mold, c) injecting melted raw material into said cylindrical raw material, and blowing said melted raw material into said reinforcement members so as to form a first stage product of the air spoiler; and, d) performing a surface finishing process for said first stage product of the air spoiler so as to obtain an end-product of the air spoiler.

7. The method for manufacturing an air spoiler for an automotive vehicle according to claim 6, wherein said engagement reinforcing member is an adhesive tape which is adhered in ring-shape on the inside wall surface of said main body portion of said reinforcement member.

8. The method for manufacturing an air spoiler for an automotive vehicle according to claim 6 , wherein said engaging reinforcement member is a predetermined plastic primer which is injected to the inside of the main body portion of said reinforcement member.

9. A method for manufacturing an air spoiler for an automotive vehicle utilizing a core mold for forming an upper portion of the air spoiler and a cavity mold for forming a lower portion of the air spoiler, comprising the steps of: positioning a plurality of separate reinforcement members in said cavity mold at positions corresponding to areas where the air spoiler is fitted on said automotive vehicle; tightly closing said core mold and said cavity mold and centering around a mold mating surface; supplying cylindrical raw material for forming the air spoiler between the core mold and the cavity mold; forming air-blowing holes through said cylindrical raw material; air-blowing said cylindrical raw material into said core mold and said cavity mold by simultaneously engaging said cylindrical raw material to engagement portions of said reinforcement members and injecting said cylindrical raw material into a main body portion of said reinforcement members; and, slowly opening said core mold and said cavity mold.

10. A method for manufacturing an air spoiler according to claim 9, further comprising exhausting air from said reinforcement members.

11. A method for manufacturing an air spoiler according to claim 9, further comprising the step of removing surface defects from said air spoiler.

\section{Manufacturing images of spoilers}

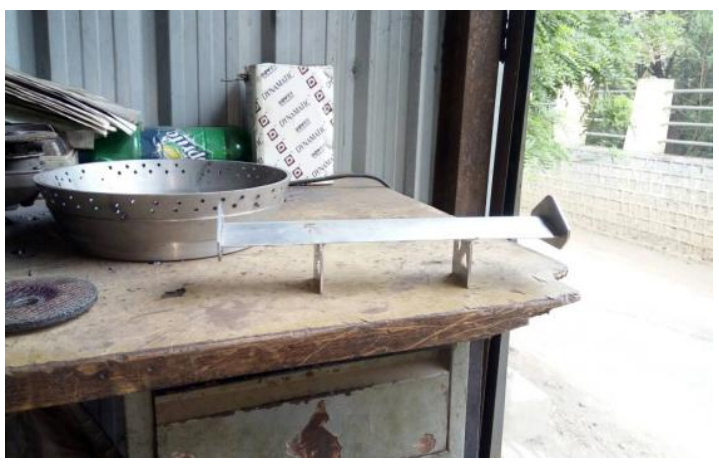




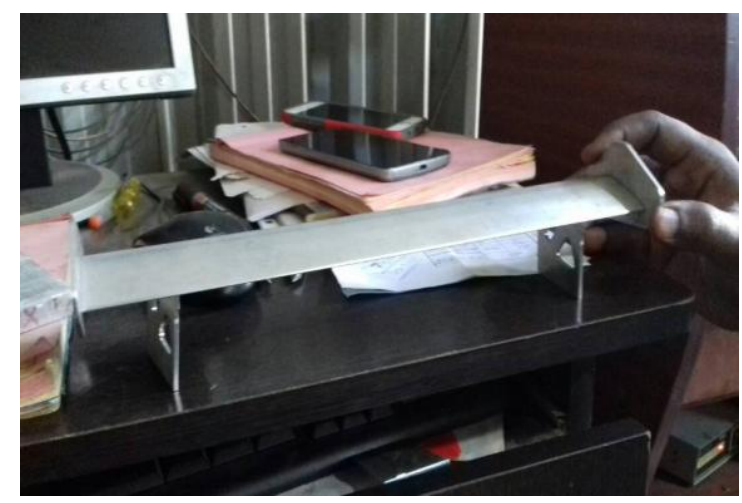

\section{CONCLUSION}

In this project, a car rear spoiler is designed and modeled in 3D modeled software CREO by different models of spoilers (NACA 4412 \& NACA 6409) and analysis done by ANSYS software at different speeds (70, 90 \& $110 \mathrm{~km} / \mathrm{hr})$. By observing the CFD analysis results the pressure drop and velocity, drag force, lift force and mass flow rate will be increases by increasing the speeds. The drag forces decreases at modified model by the speed $70 \mathrm{~km} / \mathrm{hr}$. lift force will increases at modified model by the speed $110 \mathrm{~km} / \mathrm{hr}$.

So it can be concluded the lift force more for modified model by the speed at $110 \mathrm{~km} / \mathrm{hr}$ so modified model of spoiler is better model.

\section{REFERENCES}

1. A Numerical Study On Rear-spoiler Of Passenger Vehicle Xu-xia Hu, Eric T.T. Wong.

SPOILERS' rohit milind rishi Student Department of Mechanical Engineering JDIET, Maharashtra, India.

Numerical simulation of air flow over a passenger car and the influence of rear spoiler using CFD shyam p.kodaliand srinivas bezavada

Ridhwan Bin Che Zakem, Aerodynamics of Aftermarket Rear Spoiler, Malaysia Pahang University, 2008.

5. Inchul Kim, Huailei Chen and Roger C. Shulze, "A Rear Spoiler of a New Type that Reduces the Aerodynamic Forces on a Mini-Van", SAE Technical Paper Series, 2006-01-1631. 